ISBN 978-981-14-1684-2

Proceedings of 2019 the 9th International Workshop on Computer Science and Engineering

(WCSE 2019 SUMMER)

Hong Kong, 15-17 June, 2019, pp. 897-901

doi: $10.18178 /$ wcse. 2019.06 .134

\title{
Low-Cost Wave Profiling Device for Transverse Wave Characterization
}

\author{
Conrado F. Ostia, Jr. ${ }^{1+}$, Allen Abarquez ${ }^{1}$, Kim Barlongo ${ }^{1}$, Marc Joseph Ferrer ${ }^{1}$, Jose Villa ${ }^{1}$ and \\ Glenn Magwili ${ }^{1}$ \\ ${ }^{1}$ School of Electrical, Electronics and Computer Engineering, Mapúa University, Philippines
}

\begin{abstract}
This study focused on the design and construction of a Low-Cost Device with Data Logger using Arduino Microcontroller capable of profiling the transverse wave. Series of low-cost potentiometers were used as sensor in profiling the transverse wave. The device resembled a long boat with two floaters on each segment that is deployed on the pool and sea. Results showed that the integrated measurement system was able to give statistically accurate data. Furthermore, the final prototype was able to profile a wave in terms of its sinusoidal attributes, i.e. amplitude (height), frequency and period.
\end{abstract}

Keywords: wave profiling device, wave sensor, wave measurements, micro controller, transverse wave

\section{Introduction}

Transverse waves are sinusoidal waves vibrating perpendicular to the direction of the wave's path. It can be depicted as a sine waveform where any point in the wave is comparative to the sine of an angle. Different forces of nature are represented as transverse waves [1]. Ripples on water, sound and light are all waves incorporated with transverse waves [2], [3]. With existing technologies, harvesting energies accumulated with these waves are attainable [4], [5]. However, harnessing wave energy is not a simple process due to the unpredictable waves. Thus, the researchers are challenged to characterize the present wave that can be used for research purposes and optimization process of wave energy converters.

The main objective of the study is to develop a low-cost wave profiling device for transverse wave characterization. The specific objectives are: to test functionality of the prototype, to conduct uncontrolled environment testing, to generate a wave profile based on measured parameters, and to use Fourier analysis as mathematical justification.

The study covers a device that profiles the sinusoidal attributes of a transverse wave in an uncontrolled environment. It will use a sensor that detects the up and down motion of the wave and automatically transfers the data to the computer. The height $(\mathrm{H})$ and period $(\mathrm{T})$ of the transverse wave will be displayed through the computer. Sideways motion of the wave will be a limitation since the researchers only considered rippling of the water surface and attenuation. Deploying the device in a stormy weather is another limitation to be considered. Further studies may include three dimensional motions of the waves for further accuracy of data and simulation.

\section{Methodology}

\subsection{Conceptual framework}

Fig. 1 below shows the conceptual framework of the wave profiler. The system used potentiometers as angle detectors. Pre-set values from potentiometer calibration acted as data set for input values. Inputs were

\footnotetext{
+ Corresponding author. Tel.: + 639273206191

E-mail address: cfostia@mapua.edu.ph
} 
the changes in resistance and converted to angles based on the recorded data set. Angles detected by the succeeding potentiometers were corrected based on the angle obtained at the reference. Since the reference is not fixed, relative-motion analysis is used for calculation purposes. Also, height calculation was done based on right triangle theorems. With the total length of the system as constant, the height was determined at different times using sine function given that the total length of the system as hypotenuse. Period was obtained using the frequency obtained in the Fast Fourier Transform of Matlab.

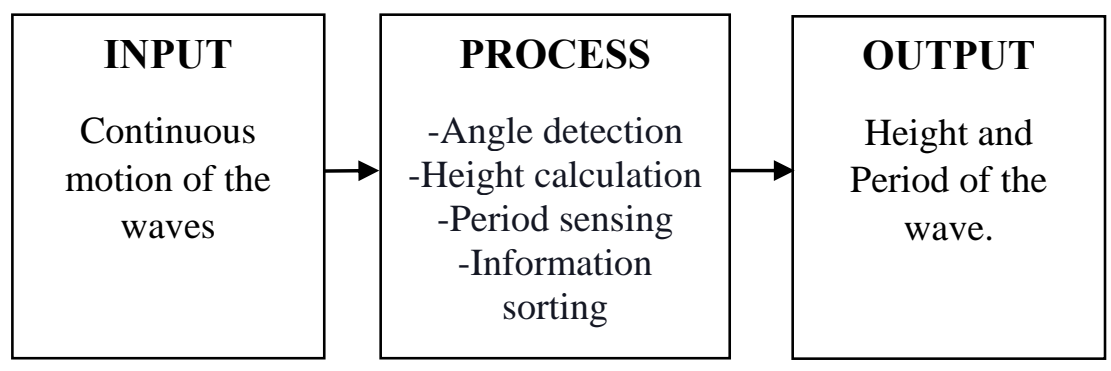

Fig. 1 Conceptual Framework of the Transverse Wave Profiler

\subsection{Design of the transverse wave profiler}

The design of the transverse wave profiler resembles a long boat as shown in Fig. 2. Ten potentiometers were joint having floaters for both sides in each segment. Each potentiometer had a lever that connected a series of potentiometer lining up from the microcontroller. The potentiometer and other electronic devices were covered in waterproof casing. Electronic devices were placed at top of the segment near the anchor.

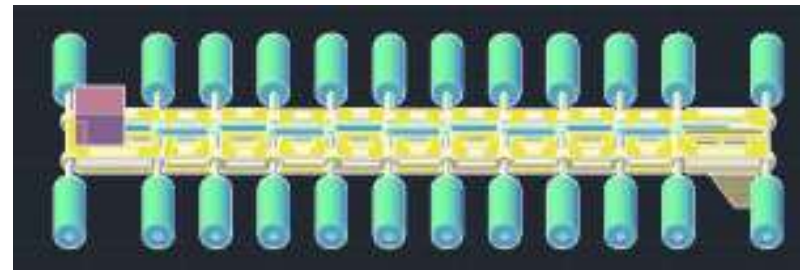

Fig. 2 Isometric View of Transverse Wave Profiler

\subsection{Controlled environment testing}

The wave profiler as shown in Fig. 3, was deployed in a controlled environment to test its functionality as shown in figure. Five trials measuring height and frequency were performed. Manual measurements using meter stick were conducted at the same time the profiler was gathering data. For statistical accuracy, twotailed t-test was carried out to compare manual and gathered data.

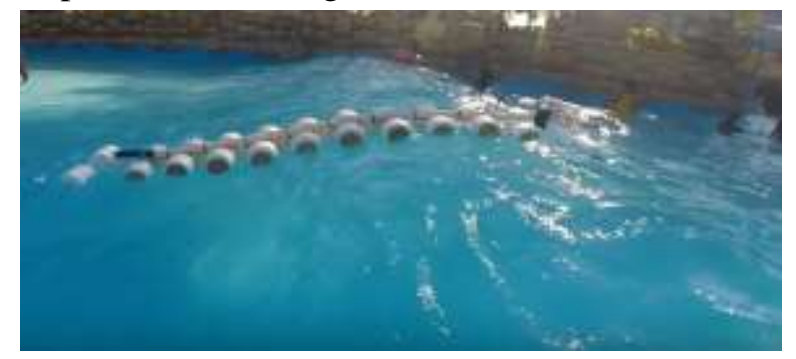

Fig. 3 Controlled Environment Testing

\subsection{Uncontrolled environment deployment}

After functionality of the wave profiler was assured, deployment to the sea was carried out as shown in figure. Five trials occurring at different locations was conducted. The wave profiler gathered data for 24 hours for the first trial, from which succeeding trials depended on the most appropriate time for deployment. All garnered data from five trials were subjected to Fast Fourier Transform (FFT) analysis to obtain a mathematical model and to compare their dominant frequencies. Fig. 4 shows the actual deployment. 


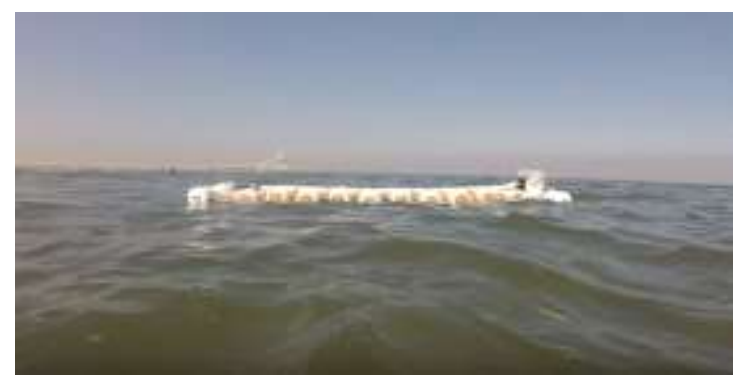

Fig. 4 Uncontrolled Environment Deployment

\section{Results and Discussion}

\subsection{Controlled environment testing}

Controlled environment testing in the wave pool resulted a maximum height of $162.06 \mathrm{~mm}$ per second. Measured values in trial 1 indicated an increase in height from an initial level of $0 \mathrm{~mm}$ and reaches a peak of $300.320078 \mathrm{~mm}$ and gradually decreased to $0.438964 \mathrm{~mm}$ showing one half of the cycle. Frequency obtained using FFT [6] was $0.1594 \mathrm{~Hz}$ which corresponds to a period of $6.2735 \mathrm{~s}$.

Obtained data were statistically accurate when compared to another set of information gathered by manual measurements as shown in Table 2. A T-stat of 1.404048 which is less than the T-critical of 2.446912 justifying the claim that "there is no significance difference between means of both manual and measured values".

\subsection{Uncontrolled environment deployment}

At Trial 1, the wave profiler was deployed to the sea $50 \mathrm{~m}$ from the shore for 24 hours. Table 3 showed only the first 0.5 seconds of data gathered to minimize the table and to show clarity over the waveforms (Figure 5). Maximum height measured by the prototype is $73.2151 \mathrm{~mm}$ occurred at $0.3 \mathrm{~s}$ from the start of trial 1. Frequency obtained after FFT analysis resulted at $1.106 \mathrm{~Hz}$ which corresponds to a period of $0.9042 \mathrm{~s}$.

\subsection{Fast fourier transform analysis (FFT)}

Table 4 represents the Amplitude (A) form of the Fourier series and the function of the dominant frequency for each trial conducted on the uncontrolled environment test. First, all measured heights of each trials in table was inputted in MATLAB, Fast Fourier Transform is then performed for analysis. 'A' are the amplitude of the spikes while ' $\mathrm{w}$ ' omega can be computed using the obtained frequencies. Dominant frequencies are the spike which is the highest in amplitude.

Table 5 represents the Fourier ' $a$ ', 'b' coefficients and the function of the dominant frequency for each trial conducted on the uncontrolled environment test. The researchers used MATLAB to get the Fourier ' $a$ ', ' $b$ ' coefficients and the dominant frequency. ' $a$ ' and ' $b$ ' coefficients are obtained from these spikes with the same frequency got from the table. The fourier series equation obtained from table can be justified by manual computation using the conversion between amplitude form to equivalent form where the values should be approximately equal. The generated Fourier series is shown in Eq. 1.

$$
\begin{gathered}
f(t)=13.15 \cos (2.588 t)-12.23 \cos (2.882 t)+12.88 \cos (3.7346 t)+\ldots \\
+1.31 \cos (6.949 t)-4.27 \sin (2.588 t)-10.23 \sin (2.882 t)- \\
9.25 \sin (3.736 t)+\ldots-8.17 \sin (6.949 t)
\end{gathered}
$$

Table 1: Controlled environment testing from trial 1.

\begin{tabular}{|c|c|}
\hline Time (s) & Height (mm) \\
\hline 0 & 0 \\
\hline 0.1 & 8.5887 \\
\hline 0.2 & 31.5264 \\
\hline 0.3 & 46.2845 \\
\hline 0.4 & 51.735 \\
\hline 0.5 & 73.7426 \\
\hline Period (s) & Frequency (Hz) \\
\hline 6.2735 & 0.1594 \\
\hline
\end{tabular}


Table 2: Two tailed t-test.

\begin{tabular}{|c|c|c|}
\hline \multirow{2}{*}{ Trial } & \multicolumn{2}{|c|}{ Height (mm) } \\
\cline { 2 - 3 } & Measured & Gathered \\
\hline $\mathbf{1}$ & 304.8 & 300.32 \\
\hline $\mathbf{2}$ & 304.8 & 302.72 \\
\hline $\mathbf{3}$ & 317.48 & 301.36 \\
\hline $\mathbf{4}$ & 304.8 & 292.37 \\
\hline $\mathbf{5}$ & 292.09 & 295.98 \\
\hline T-Stat & \multicolumn{2}{|c|}{1.404048} \\
\hline T Critical two-tail & \multicolumn{2}{|c|}{2.46912} \\
\hline
\end{tabular}

Table 3: Uncontrolled environment deployment from trial 1.

\begin{tabular}{|c|c|}
\hline Time (s) & Height $(\mathbf{m m})$ \\
\hline 0 & 0 \\
\hline 0.1 & 59.4495 \\
\hline 0.2 & 69.4411 \\
\hline 0.3 & 73.2151 \\
\hline 0.4 & 70.2746 \\
\hline 0.5 & 62.1763 \\
\hline Period (s) & Frequency (Hz) \\
\hline 0.9042 & 1.106 \\
\hline
\end{tabular}

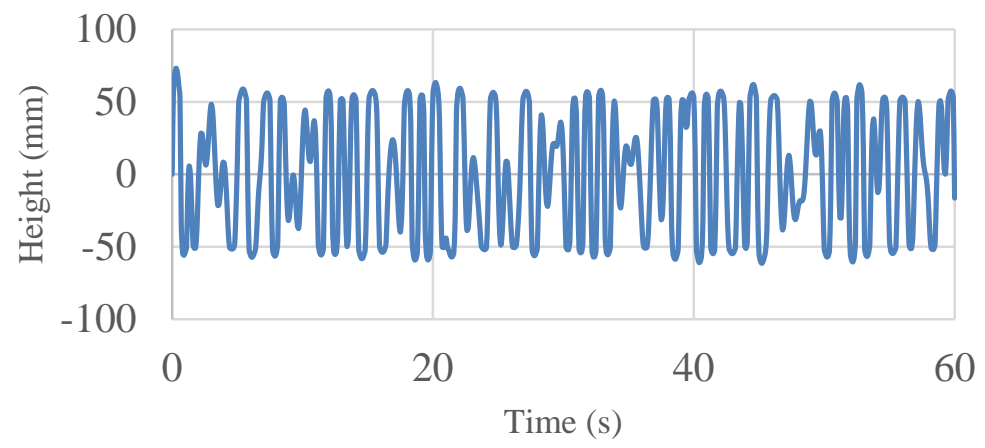

Fig. 5 Generated Waveform for $50 \mathrm{~m}$.

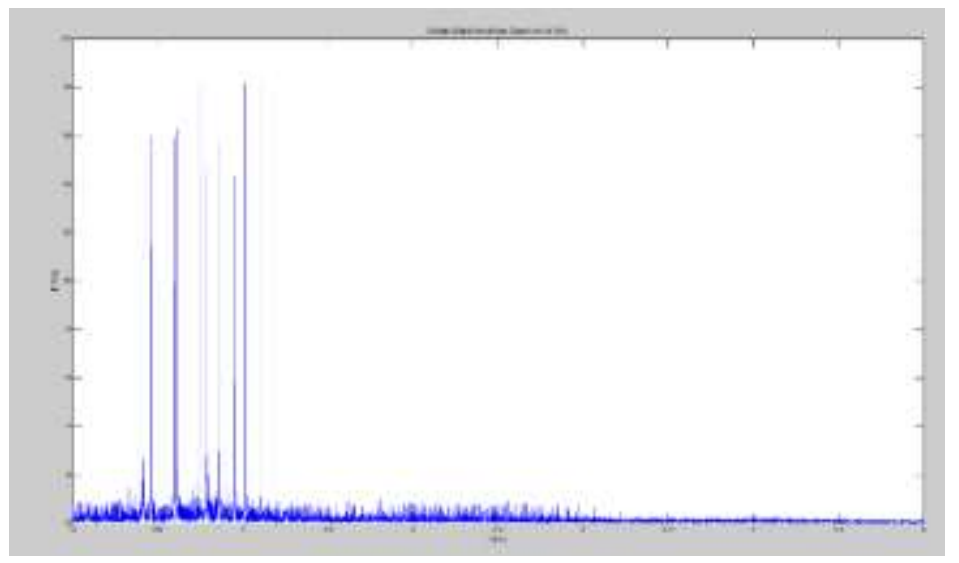

Fig. 6 Fast Fourier Transform for Trial 1 (50 m ). 
Table 4: Fast fourier transform analysis from trial 1.

\begin{tabular}{c|c|c|c|}
\hline \multicolumn{2}{c|}{ Amplitude (A) } & \multicolumn{2}{c|}{ w } \\
\hline $\mathrm{A}_{1}$ & 13.83 & $\mathrm{w}_{1}$ & 2.588 \\
\hline $\mathrm{A}_{2}$ & 15.94 & $\mathrm{w}_{2}$ & 2.882 \\
\hline $\mathrm{A}_{3}$ & 15.86 & $\mathrm{w}_{3}$ & 3.7346 \\
\hline$\ldots$ & $\ldots$ & $\ldots$ & $\ldots$ \\
\hline $\mathrm{A}_{10}$ & 18.22 & $\mathrm{w}_{10}$ & 6.949 \\
\hline \multicolumn{2}{c|}{ Frequency (Hz) } & \multicolumn{2}{c|}{ Period (s) } \\
\hline \multicolumn{2}{c|}{1.106} & \multicolumn{2}{c}{00415} \\
\hline
\end{tabular}

Table 5: Fast fourier transform coefficients.

\begin{tabular}{|l|c|c|c|c|}
\hline \multicolumn{2}{|c|}{ a coefficients } & \multicolumn{2}{c|}{ b coefficients } & w \\
\hline $\mathrm{a}_{1}$ & 13.15 & $\mathrm{~b}_{1}$ & -4.27 & 2.588 \\
\hline $\mathrm{a}_{2}$ & -12.23 & $\mathrm{~b}_{2}$ & -10.23 & 2.882 \\
\hline $\mathrm{a}_{3}$ & 12.88 & $\mathrm{~b}_{3}$ & -9.25 & 3.7346 \\
\hline$\ldots$ & $\ldots$ & $\cdots$ & $\ldots$ & $\ldots$ \\
\hline $\mathrm{a}_{10}$ & 1.31 & $\mathrm{~b}_{10}$ & -18.17 & 6.949 \\
\hline \multicolumn{3}{|c|}{ Frequency (Hz) } & \multicolumn{3}{|c|}{ Period (s) } \\
\hline \multicolumn{3}{|c|}{1.106} & 0.90415 \\
\hline
\end{tabular}

\section{Conclusions}

The researchers were able to test the functionality of the device by deploying on controlled environment. It was observed that a peak value of $300.320078 \mathrm{~mm}$ was measured in the wave pool and a height of $304.8 \mathrm{~mm}$ was measured manually. Using t-test statistical analysis, it showed that the wave measured values and the manual measurement at controlled environment have a T-stat of 1.404048 which is less than the critical $\mathrm{T}$ value of 2.446912 therefore, it can be concluded that both values are statistically equal.

\section{Acknowledgements}

The authors wish to thank the School of Electrical, Electronics and Computer Engineering of Mapúa University for the assistance, to our love ones for their encouragement and support, and above all, to the almighty God for giving us the wisdom and courage to finish this study.

\section{References}

[1] Niu, X.. Modeling and Design Analysis of a Permanent Magnet Linear Synchronous Generator. Engineering System Design Lab University of Illinois. Urbana-Champaign, August 2013.

[2] Lotha, G., et al., Transverse wave: Longitudinal and Transverse Wave Motion, November 4, 2016. [Online]. Available: https://www.britannica.com/science/transverse-wave. [Accessed: February 2, 2019]

[3] H. A. Young and R. A. Freedman, University Physics with Modern Physics, 13th ed.. San Francisco CA: Pearson Education, Inc., 2016.

[4] J. Broeders, R. W. Pascal, C. Cresens, E. M. Waugh, C. L. Cardwell and M. J. Yelland, "Smart electronics for high accuracy wave height measurements in the open ocean," OCEANS 2016 MTS/IEEE Monterey, Monterey, CA, 2016, pp. 1-5.

[5] A. E. Barakaz and A. E. Marjani, "Analysis of Aero-hydrodynamic Equations Inside an OWC Device for Wave Energy Conversion," 2017 International Renewable and Sustainable Energy Conference (IRSEC), Tangier, 2017, pp. 1-6.

[6] Gustafson, G. B., "Periodic Boundary Conditions and the Complete Fourier Series," Analytical and Computational Methods of Advanced Engineering Mathematics, pp. 492-501, 1998. 